\title{
The Knee Complex Considered as Perceptual Systems
}

\section{Wangdo Kim*}

Ingeniería Mecánica, Universidad de Ingenieria y Tecnologia - UTEC, Lima 15063, Perú

*Corresponding Author: Wangdo Kim, Ingeniería Mecánica, Universidad de Ingenieria y Tecnologia - UTEC, Lima 15063, Perú

Received date: 17 June 2021; Accepted date: 24 June 2021; Published date: 30 June 2021

Citation: Kim W. The Knee Complex Considered as Perceptual Systems. J Med Case Rep Case Series 2(6):

https://doi.org/10.38207/jmcrcs20210074

Copyright: (C) 2021 Wangdo Kim. This is an open-access article distributed under the terms of the Creative Commons Attribution License, which permits unrestricted use, distribution, and reproduction in any medium, provided the original author and source are credited.

\begin{abstract}
Proprioception, considered as the obtaining of information about one's own action, does not necessarily depend on proprioceptors. At the knee joint, perceptual systems are active sets of organs designed to reach equilibrium through synergies-the specific mechanoreceptors in the knee predominantly located on the cruciate ligaments. The terminals are of many sorts, not easy to distinguish. There are free nerve endings, the bare branches of the afferent fibers that can spread out, spray, or cluster in basket-like or flower-like arrangements. These are extremely dense. To call them "receptors" may sound strange, but the fact is that the naked peripheral nerve cell is excited by squeezing it, and it is therefore receptive. Ligaments have significant input to sensation and synergistic activation of muscles. The ligamento-muscular reflex is responsive to mechanical stimulation techniques within the scope of practice for physiotherapy. Utilizing specific manual techniques can have positive effects in attenuating proprioceptive and kinesthetic neurological deficits.
\end{abstract}

Keywords: The ligamento-muscular reflex; Mechanoreceptors; Gibson's affordance; The principle of least action; Tensegrity’s structure; Instantaneous knee axis (IKA); The knee complex; Gibson’s ecological mechanics

\section{Distributions of mechanoreceptors in the knee complex}

For the last 20 years, orthopedic surgeons have been interested in the cruciate ligaments, mechanical and structural stabilizers of the knee joint, and sensory structures. The review [6] indicates that the PCL may serve as a 'mechanical stabilizer' of the knee joint and probably has a significant 'sensory function' that should be considered when dealing with injuries. The posterior cruciate ligament provides $95 \%$ of the restraining force to a posterior tibial displacement is significantly stronger than the other knee ligaments, and sensory nerve endings are located in the tibia and femoral bone insertions.

The ACL is highly susceptible to failure during athletic activities and slip-fall events (Howell 1998). The goal of ACL reconstruction surgery is to rebuild the ligament attachments as closely as possible to the native anatomy in order to restore pre-injury knee function and normal proprioception in the affected knee (Behrend, Giesinger et al. 2017).

The joints are exploratory sense organs, but they are also performative motor organs; that is to say, the equipment for feeling is anatomically the same as the equipment for doing [3]. Considering the importance of the sensory function of the joint structure, it would seem sensible to minimize the sensory damage of the joint whenever operative treatment is necessary (Johansson, Sjölander, et al. 1991).

The knee joint has a wide range of movements that are supported by the surrounding ligaments, tendons, and muscles. Kennedy et al. described mechanoreceptors and nerve fibers in the posterior cruciate ligament (PCL), an anterior cruciate ligament (ACL), meniscus, and capsular structures [7]. These mechanoreceptors are found all through the knee joint, but in particular, they are associated with the following parts and organs. The notable thing which all these parts and organs have in common is that they are mobile. But they are mobile in different mechanical ways. The knee joints move only by their own instantaneous knee axes and have a fixed set of angular positions [11]. The ligaments are tensioned by isometric positioning at the attachments [19]. The muscles can only contract or relax relative to their antagonists, with varying amounts of pull at varying lengths. The other tissues have their own emotions and states. The information picked up by the receptors in any part of the knee must be information about the particular motion or state of that part, not simply information about what part it is [3]

The mechanical role of the anterior and posterior cruciate ligaments in the passive and functional stability of the knee joint has been well documented. Both these knee joint ligaments contain Ruffini, Pacinian, Golgi and free nerve endings with different capabilities of providing the central nervous system with information regarding movement and position as well as chemical events [6]. The ligaments were considered, over several centuries, as the major restraints of the joints, keeping the associated bones in position and preventing instability, e.g., their separation from each other and/or mal alignment. According to M. Solomonow, ligaments are also major sensory organs, capable of monitoring relevant kinesthetic and proprioceptive data; Excitatory and inhibitory reflex arcs from sensory organs within the ligaments recruit/de-recruit the musculature to participate in maintaining joint stability as needed by the movement 
type performed; The synergy of the ligament and associated musculature allocates prominent role for muscles in maintaining joint stability [17].

The existence of this reflex indicates that the cruciate ligaments have an afferent function, which influences knee dynamics [15]. Researchers basically used the model of isolated ligament injuries [5]. However, cruciate ligaments cross each other, revealing concomitant injuries (combined), thereby manifesting complex sensory-motor disorders. The structure of tendons and ligaments relates to their function. Hence the tissue matrix array reflects the tissues' biomechanical roles. Tendons transmit muscle contraction strength to bone, whereas ligaments transmit forces across bones. Unlike tendons aligned in one direction, ligaments are aligned in a web to keep the joint stable in many different positions (Figure 1(a)). In this review, we report an alternative approach based on the understanding of knee perceptual subsystems [9] to guide physiotherapies in the design/assessment of knee reconstruction strategies.
The tensegrity's structure is characterized by the cartilage contact elements $\$_{4}^{\prime}$ and $\${ }_{5}^{\prime}$ while all the other elements are continuous tension elements. They are showing specific configurations having torque less connections (Figure 1) [13,8]. We introduce the conception of the knee complex, i.e., the action lines which satisfy the given condition as the tensegrity's structure is characterized by transmitting forces across the bones [10]. The kinematic possibilities of locomotion or the affordance of walking require that only the component of the resultant moment in the direction of the axis of the knee has to vanish for equilibrium [4], and it takes up a position of stationary potential energy [16]. Not included in Figure 1(b) is the role played by the gravity-force and inertial forces. Supposing the knee complex to be in equilibrium under the influence of a system of constraint forces along the five strings together with the actions of gravity and inertia, then the sum total of reaction must be augmented by the latter forces into the original knee complex.

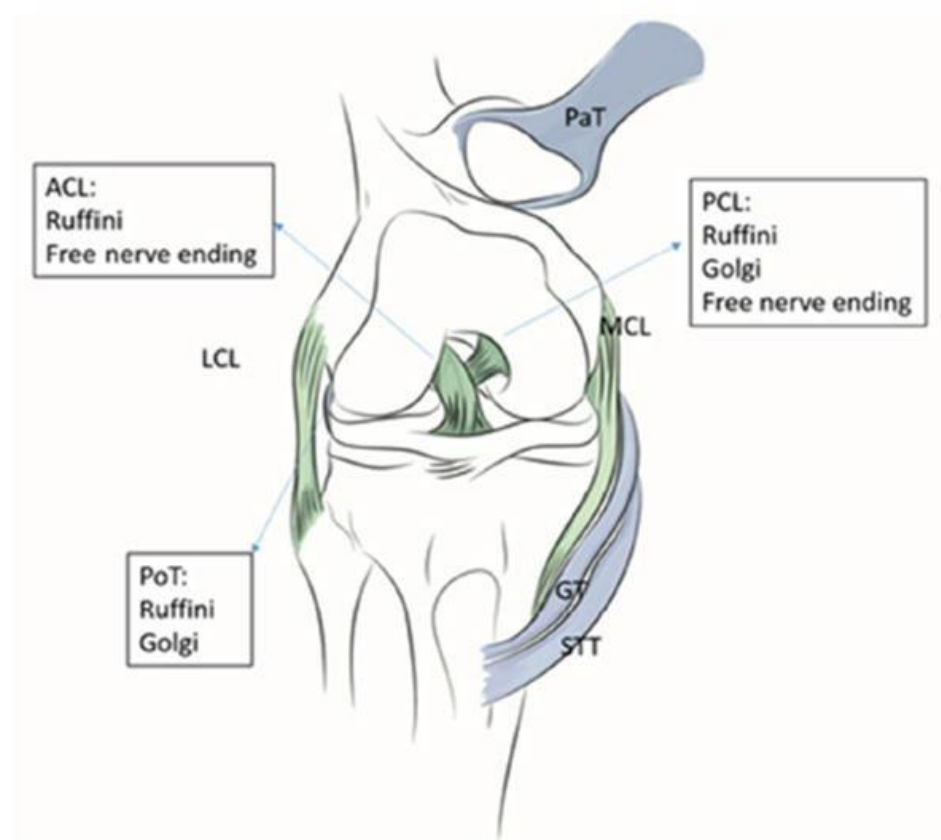

(a)

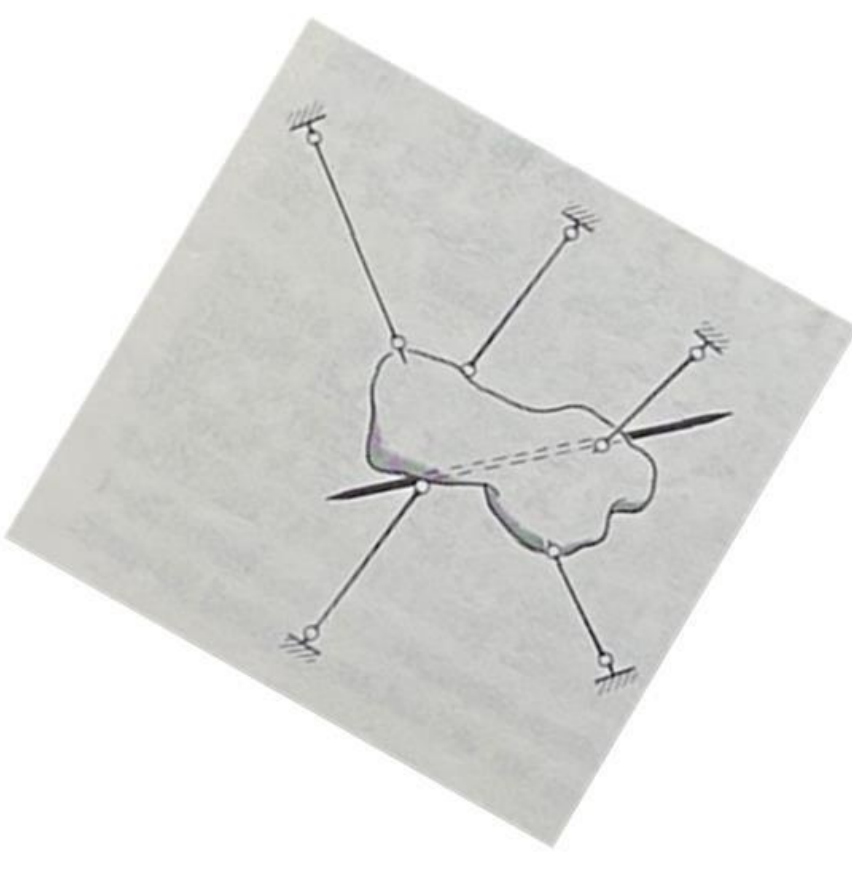

(b)

Figure 1 (a) : A schematic drawing of the ligaments (in green) and tendons (in blue) studied; PCL, ACL and PoT were rich in mechanoreceptors (PaT: patellar tendon, STT: semitendinosus tendon, LCL: lateral collateral ligament, PoT: popliteal tendon, GT: gracilis tendon, PCL: posterior cruciate ligament, ACL: anterior cruciate ligament). The original schematic and images were published previously [1] and are used by the permission of Dr. Haluk Çabuk.

(b) A body suspended on five taut strings moves with one degree of freedom. The ISA for small oscillation is shown. The original anatomic schematics and lines of action were published previously $[\mathbf{1 2 , 1 4 , 2 ]}$ and are used with the permission of Professor Michele Conconi. The video is available:

\section{https://drive.google.com/file/d/18_YtszzT3_IvNIken5uxObj4jmSd0Zs/view?usp=sharing}

\section{The ligamento-muscular reflex}

Take the \$, the instantaneous knee axis (IKA), from the knee complex, which defines the freedom, and five taut strings forming the knee complex, which is the system of reactions of the constraints. Decompose the given muscle force into components on these six lines $\$_{i}, i=1, . ., 6$. The components $\$_{\mathrm{i}}^{\prime}$ are neutralized by the reactions of the constraints and may be discarded, while the remainder must compound into torque on the given system, the IKA. Given muscle forces can, in general, be resolved into two parties - one on a degree of freedom, and the others on a taut string tension in ligaments and cartilage contacts in the knee complex. Therefore, muscular activity elicited by this reflex allows muscles and ligaments to work together in maintaining joint stability.

The ligamento-muscular reflex states this result in a somewhat different manner. Throughout the knee complex run two forces: the one is mechanically operated by the principle of least action, working 
on all parts of the body in the same way that it acts on any similar combination of weights, levers, and supports [16]; the other is the living force exerted by the neuromuscular mechanism. The muscle task is to move the bones, balance them at six constraints in the joint such that no work results along the axis of the knee, and transfer their weights as directly as possible. They should be used for this purpose and not to do the work assigned to the bones. This muscular action, directed by the ligamento-muscular reflex nervous system, performs the patterns of only the component of the resultant moment in the direction of the axis of the knee being zero [10]. If muscles are called upon to lift and to hold weights unnecessarily instead of to move bones in a balanced relationship, such action violates their

\section{Acknowledgments}

The author acknowledges the support from the MIT-Peru UTEC Seed Fund awarding the project titled "Development of Proper Tunnel Syndrome Placement Device to Avoid Impingement." The experimental data used for validation were provided by the "Grand

\section{References}

1. Çabuk, H. and F. Kuşku Çabuk (2016) Mechanoreceptors of the ligaments and tendons around the knee. Clinical Anatomy 29(6) 789-795.

2. Conconi M, Sancisi N, Castelli VP (2019) The Geometrical Arrangement of Knee Constraints That Makes Natural Motion Possible: Theoretical and Experimental Analysis. Journal of Biomechanical Engineering 141(5): 051001.

3. Gibson JJ (1966) The senses considered as perceptual systems. Boston, Houghton.

4. Gibson, JJ (1979) The ecological approach to visual perception, Houghton Mifflin.

5. Johnson P, Mitchell SM, Görtz S (2018) Graft Considerations in Posterior Cruciate Ligament Reconstruction. Current Reviews in Musculoskeletal Medicine 11(3): 521-527.

6. Katonis P, Papoutsidakis A, Aligizakis A, Tzanakakis G, Kontakis G, et al. (2008) Mechanoreceptors of the posterior cruciate ligament. Journal of International Medical Research 36(3): 387-393.

7. Kennedy JC, Alexander IJ, Hayes KC (1982) Nerve supply of the human knee and its functional importance. The American journal of sports medicine. 10(6): 329-335.

8. Kim W (2019) Tibial Femoral Tunnel for Isokinetic Graft Placement Based on a Tensegrity Model of a Knee. Knee Surgery-Reconstruction and Replacement, IntechOpen.

9. Kim W (2020) The Knee Proprioception as Patient-Dependent Outcome Measures within Surgical and Non-Surgical Interventions. Proprioception, IntechOpen.

10. Kim W, Araujo D, Kohles SS, Kim SG, Alvarez Sanchez HH (2021). Affordance-Based Surgical Design Methods relation with the nervous system, as the organic sensations then sent to it are not designed to induce the appropriate reflexes.

This review paper suggests that "therapists might be encouraged to aim their soft tissue technique to a lesser area where there is known to be a high density of mechanoreceptors." In our dynamic organism, correlation is accomplished in two interrelated ways: mechanical changes through direct transmission of pressure or tension and organic changes through the excitation of living tissue. The effect of the neuromuscular force is to move bones about, while the effect of the mechanical force is to move them in accordance with the principles of natural balance [18].

Challenge Competition to Predict In Vivo Knee Loads" as part of the Symbiosis project funded by the US National Institutes of Health via the NIH Roadmap for Medical Research (Grant \# U54 GM072970).

Considering Biomechanical Artifacts. Ecological Psychology. 33(1): 57-71.

11. Kim W, Choi Y, Lee H (2016) Observations on the Knee Functional Axis During Active Movements. SM Musculoskelet Disord 1(1): 1004.

12. Kim W, Espanha MM, Veloso AP, Araújo D, João F, et al (2013) An informational algorithm as the basis for perception-action control of the instantaneous axes of the knee. Journal of novel physiotherapies 3(1): 127.

13. Kim W, Park A (2018) Topological Space of the Knee Tensegrity System. Biomedical Journal 2: 3.

14. Kim W, Veloso AP, Araújo D, Kohles SS (2014) Novel computational approaches characterizing knee physiotherapy. Journal of Computational Design and Engineering 1(1): 55-66.

15. Krogsgaard MR, Dyhre-Poulsen P, Fischer-Rasmussen T (2002) Cruciate ligament reflexes. Journal of Electromyography and Kinesiology 12(3): 177-182.

16. Lanczos C (2012) The variational principles of mechanics. Courier Corporation.

17. Solomonow M (2006) Sensory-motor control of ligaments and associated neuromuscular disorders. Journal of Electromyography and Kinesiology 16(6): 549-567.

18. Todd ME, Brackett EG (1937) The Thinking Body: A Study of the Balancing Forces of Dynamic Man. Incorporated.

19. Trus P, Peterman J, Gotzen L (1994) Posterior cruciate ligament (PCL) reconstruction-an in vitro study of isometry. Knee Surgery, Sports Traumatology, Arthroscopy 2(2): 104-106. 